\title{
Continuous-variable quantum erasure correcting code
}

Lassen, Mikael Østergaard; Sabuncu, Metin; Huck, Alexander; Niset, J.; Cerf, N.; Leuchs, G.; Andersen, Ulrik Lund

Published in:

2010 Conference on Lasers and Electro-Optics (CLEO) and Quantum Electronics and Laser Science

Conference (QELS)

Publication date:

2010

Document Version

Publisher's PDF, also known as Version of record

Link back to DTU Orbit

Citation $(A P A)$ :

Lassen, M. Ø., Sabuncu, M., Huck, A., Niset, J., Cerf, N., Leuchs, G., \& Andersen, U. L. (2010). Continuousvariable quantum erasure correcting code. In 2010 Conference on Lasers and Electro-Optics (CLEO) and Quantum Electronics and Laser Science Conference (QELS) (pp. 1-2). IEEE.

\section{General rights}

Copyright and moral rights for the publications made accessible in the public portal are retained by the authors and/or other copyright owners and it is a condition of accessing publications that users recognise and abide by the legal requirements associated with these rights.

- Users may download and print one copy of any publication from the public portal for the purpose of private study or research.

- You may not further distribute the material or use it for any profit-making activity or commercial gain

- You may freely distribute the URL identifying the publication in the public portal 


\title{
Continuous-Variable Quantum Erasure Correcting Code
}

\author{
Mikael Lassen $^{1, *}$, Metin Sabuncu ${ }^{1,2}$, Alexander Huck ${ }^{1}$, Julien Niset ${ }^{3,4}$, Nicolas Cerf ${ }^{3}$, Gerd Leuchs ${ }^{2,5}$ and Ulrik \\ L. Andersen ${ }^{1}$ \\ ${ }^{1}$ Department of Physics, Technical University of Denmark, Building 309, 2800 Lyngby, Denmark \\ ${ }^{2}$ Max Planck Institute for the Science of Light, Gunther Scharowsky Strasse 1, 91058 Erlangen, Germany \\ ${ }^{3}$ Quantum Information and Communication, Ecole Polytechnique, CP 165, Universite Libre de Bruxelles, 1050 Brussels, Belgium \\ ${ }^{4}$ Department of Physics, Hunter College of CUNY, 695 Park Avenue, New York, NY 10065 \\ ${ }^{5}$ University Erlangen-Nürnberg, Staudtstrasse 7/B2, 91058 Erlangen, Germany. \\ *e-mail address: mlassen@fysik.dtu.dk
}

\begin{abstract}
We experimentally demonstrate a continuous variable quantum erasure-correcting code, which protects coherent states of light against complete erasure. The scheme encodes two coherent states into a biparty entangled state, and the resulting 4-mode code is conveyed through 4 independent channels that randomly erases the signal. We show experimentally that the transmitted state can be corrected by performing a syndrome measurement followed by a corrective transformation.

C2010 Optical Society of America
\end{abstract}

OCIS codes: (270.0270) General; (270.5585)

\section{Introduction}

Quantum information processing relies on the robust and faithful transmission, storage and manipulation of quantum information [1]. However, since errors are inherent to any realistic implementation, the future of quantum information systems strongly relies on the ability to detect and correct for these errors. Quantum error code corrections (QECC) were first discovered for discrete variable qubit systems [2,3] and only a few experimental implementations demonstrating correction of corrupted qubit states have been carried out.

We consider a scheme to eliminate these errors in a continuous variable (CV) quantum channel [4]. The error correction can be deterministic if the location of the error is known and only a single channel has been affected. If the location of the erasure is unknown and more than one channel has been corrupted, the signal can be recovered probabilistically where the transmitted state is either kept of discarded depending on whether an error was detected in the syndrome measurement. We have experimentally demonstrated both strategies and obtained transmission fidelities beyond what is possible by classical approaches.

\section{Experimental Realization of the QECC}

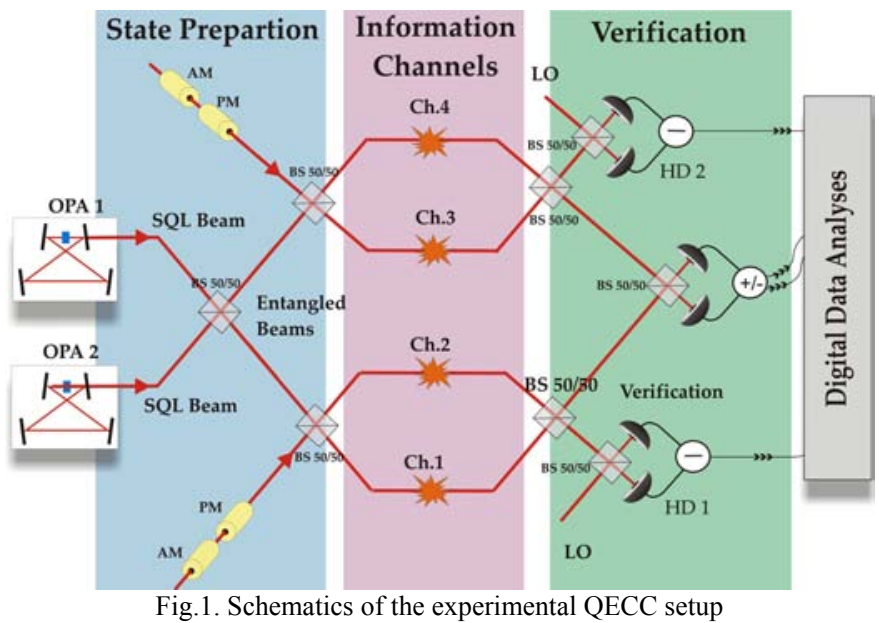

The schematics of our setup are depicted in Fig. 1. The scheme encodes two coherent states into a bi-party entangled state, and the resulting 4-mode code is conveyed through 4 independent channels that randomly erases the signal. The key elements are the entanglement source, the quantum state preparation stage, the information channels and the verification stage. The entanglement is produced through the interference of two Gaussian, single-mode amplitude squeezed states generated by two optical parametric oscillators. The coherent states (the quantum information) are 


\section{JThE25.pdf}

generated at a sideband frequency of $5.5 \mathrm{MHz}$ using amplitude- and phase-modulators. The quantum information network consists of two Mach-Zehnder interferometers. The coherent states are measured after transmission by performing homodyne detections, HD1 and HD2, and the syndrome measurement (SM) is performed by heterodyne detection and detects whether an erasure has occurred.
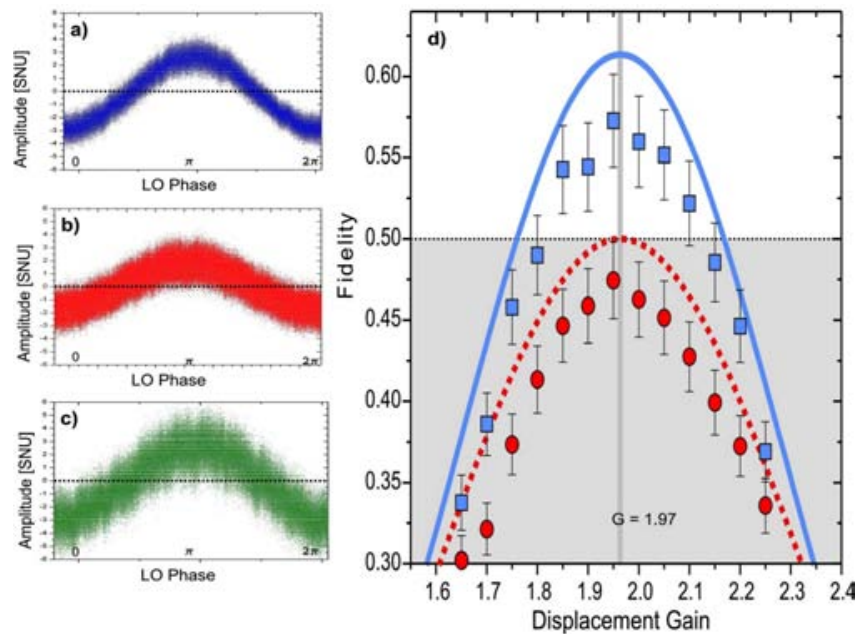

FIG. 2: Results of the deterministic QECC protocol.

The deterministic protocol relies on the fact that one can correct the losses provided that one can monitor the occurrence of erasures. For example if Ch.2 is lost, the coherent state will be attenuated and polluted by quantum noise. However, since this noise is exactly correlated with the other half of the entanglement pair, we can partly recover the coherent state by amplifying the measurement with a gain of 2 , where the second output port of the Mach-Zehnder interferometer is used as the idler input of the amplifier. In the work by J. Niset et al. [4] it was shown that the fidelity with unity gain is given by: $F=1 /\left(1+\mathrm{e}^{-2 r}\right)$, where $\mathrm{e}^{-2 \mathrm{r}}$ correspond to the measured degree of two-mode squeezing.

Fig. 2a shows a scan of the quantum mechanical oscillator comprising the coherent state quantum information of the input state. Fig. $2 \mathrm{~b}$ illustrates the measurements at the homodyne detector HD1 after the fourmode state has been transmitted through the channel with erasure on channel 2 . The state is clearly seen to be corrupted as the first and second moments of the quantum oscillator are significantly changed. However, by using the measurement outcomes of the SM to appropriately displace the transmitted state with the displacement gain, G, the quantum state is partially recovered as shown qualitatively in Fig. $2 \mathrm{c}$ for $\mathrm{G}=1.97$. Based on the measurements presented above, the fidelities are computed for various gains and the results are depicted by the blue squares in Fig. 2e. A maximum fidelity of about 0.57 is obtained which clearly surpasses the classical benchmark of 0.50 . Similar fidelities are achieved for the erasure of channel 1, whereas fidelities close to unity are obtained when channel 3 or 4 are blocked. Measurements for which the two-mode squeezed state was replaced by vacuum are also carried out for different displacement gains. The resulting fidelities are depicted in Fig. 2e by the red circles, and they nicely illustrate the need for entanglement.

In this contribution we will also present results for a probabilistic scheme, where the corrupted states are probabilistically corrected. Using this scheme, the stringent channel conditions mentioned above can be relaxed; we do not need to know the occurrence and location of the erasure. For this protocol is it also evident that the use of the entanglement-based code further increases the fidelity for all evaluated error probabilities.

\section{References}

[1] Nielsen, M. A., and Chuang, I. L., Quantum Computation and Quantum Information, Cambridge University Press (2000).

[2] Shor, P. W., Scheme for reducing decoherence in quantum computer memory, Phys. Rev. A 52, R2493 (1995).

[3] Steane, A. M., Error Correcting Codes in Quantum Theory, Phys. Rev. Lett. 77, 793 (1996).

[4] Niset, J., Andersen, U. L., and Cerf, N. J., Experimentally Feasible Quantum Erasure-Correcting Code for Continuous Variables, Phys. Rev. Lett. 101, 130503 (2008) 